\title{
Lipodorsoabdominoplastias: 10 anos de experiência
}

\author{
Lipodorseabdominoplasty: 10 years of experience
}

Trabalho realizado no Serviço de Cirurgia Plástica da Santa Casa de Misericórdia de Goiânia, Goiânia, GO, Brasil.

Artigo submetido no SGP (Sistema de Gestão de Publicações) da RBCP.

Artigo recebido: $11 / 12 / 2009$ Artigo aceito: 5/11/2010

\begin{abstract}
RESUMO
Introdução: O presente estudo apresenta, retrospectivamente, a experiência do autor no período de setembro de 1999 a junho de 2010, relativa à lipodorsoabdominoplastia, e descreve a confecção do túnel cirúrgico xifoumbilical e a descida do retalho abdominal à região hipogástrica. Este processo cirúrgico é associado à lipoaspiração de gordura do abdome e dorso, com excisão da camada de gordura lamelar abaixo da fáscia de Scarpa. Método: As cirurgias foram realizadas no serviço de ensino da Santa Casa de Misericórdia de Goiânia e em clínica privada. Foram operadas 606 pacientes do sexo feminino, com idade média de 30 anos. Pacientes com sobrepeso e em pós-cirúrgico bariátrico não foram incluídas. Os abdomes operados caracterizavam-se como dos tipos III e V segundo a classificação de Bozola e Psillakis. Resultados: O procedimento cirúrgico de lipodorsoabdominoplastia mostrou-se seguro, observados os cuidados requeridos, não tendo ocorrido complicações graves, como embolia pulmonar, infecção, necrose de pele, perfurações de cavidades ou óbitos. Conclusão: O nível de excelência alcançado nos resultados dos procedimentos de lipodorsoabdominoplastia caracteriza-a como uma evolução natural da abdominoplastia clássica.
\end{abstract}

Descritores: Técnicas cosméticas. Lipectomia. Abdome/cirurgia.

\begin{abstract}
Background: This study presents retrospectively the author's experiences from September 1999 to June 2010, concerning lipodorseabdominoplasty, and describes tunnel surgical xifoumbilical performed and descent of the abdominal flap to the hypogastric region. This surgical procedure is associated with liposuction of fat from the abdomen and back with excision of the fat layer below the lamellar Scarpa fascia. Methods: All procedures were performed in an University of Santa Casa de Misericordia de Goiânia and in private clinic. Were operated 606 females patients, mean age 30 years. Overweight patients and postbariatric surgery were not included. The operated abdomens were characterized as types III and V according to the classification of Bozola and Psillakis. Results: The surgical procedure of the lipodorseabdominoplasty is safe, observing the care required and there weren't serious complications such as pulmonary embolism, infection, skin necrosis, perforation of cavities or deaths. Conclusion: The level of excellence achieved in the results of procedures lipodorsoabdominoplastia characterizes it as a natural evolution of classical abdominoplastia.
\end{abstract}

Keywords: Cosmetic techniques. Lipectomy. Abdomen/surgery. 


\section{INTRODUÇÃO}

O contorno corporal submete-se a um padrão estético e funcional que varia a cada época e é muito relevante em nossos dias.

Deformidades e disfunções deste contorno causam limitações nos relacionamentos afetivos e sociais das pessoas. Assim, a cirurgia do contorno corporal visa devolver-lhes a autoestima decorrente de uma imagem corporal positiva.

Em 1899, Kelly ${ }^{1}$ descreveu uma ressecção elíptica horizontal do abdome, desde então diversos autores contribuíram com suas técnicas cirúrgicas de abdominoplastia. $\mathrm{Babcok}^{2} \mathrm{fez}$ ressecções transversas e verticais, Thorek ${ }^{3}$ reduziu o nível da cicatriz, preservando o umbigo e, em 1942, descreveu a dermolipectomia invertida. Pontes ${ }^{4}$, em 1965, demonstrou a ressecção em bloco de todo o retalho hipogástrico em forma de elipse e Callia ${ }^{5}$, em 1965, a incisão hipogástrica sobre o púbis, com prolongamento laterais. Em 1967, Pitanguy ${ }^{6}$ relatou a execução de incisão baixa com extremidades para fora e para baixo e $\operatorname{Sinder}^{7}$ demonstrou a retirada do retalho inferior após o posicionamento do retalho superior do abdome, determinando a posição exata da cicatriz.

Estes e outros autores estabeleceram os princípios básicos da abdominoplastia realizada nos dias de hoje. Contudo, situações desagradáveis, como deiscências e necroses decorrentes do amplo descolamento do retalho cutâneo e ao solapamento do sistema vásculo-nervoso, eram frequentes.

Com o advento da lipoaspiração ${ }^{8}$, esta técnica passou a complementar as abdominoplastias, melhorando o contorno corporal.

Baroudi e Ferreira ${ }^{9}$ deram início à utilização de pontos no tecido subcutâneo aponeurótico, promovendo a adesão dos dois planos com a finalidade de prevenir seromas.

Avelar ${ }^{10}$ desenvolveu uma nova técnica de abdominoplastia, o sistema vascular fechado de retalho subdérmico dobrado sobre si mesmo, combinado com lipoaspiração, apresentado pela primeira vez no XXXVI Congresso Brasileiro de Cirurgia Plástica, no Rio de Janeiro, e publicado na Revista Brasileira de Cirurgia Plástica. A publicação relata a primeira vez em que a lipoaspiração foi associada à abdominoplastia, técnica que diminui a morbidade graças à preservação do sistema vásculo-nervoso.

Em trabalho anterior, apresentado no XXXVII Congresso Brasileiro de Cirurgia Plástica de Porto Alegre, em 2000, o autor ${ }^{11}$ deste artigo já relatou a confecção de túnel cirúrgico xifoumbilical que passou a permitir a plicatura dos músculos reto abdominais. Neste estudo, inicialmente realizado em laboratório de anatomia da disciplina de Técnica Operatória e Cirurgia Experimental da Faculdade de Medicina da Universidade Federal de Goiás (FM-UFG), foi possível realizar a confecção de um túnel xifoumbilical de mais ou menos 3 $\mathrm{cm}$ de cada lado da linha média, com preservação dos vasos perfurantes que irrigam o retalho abdominal lateralmente.

No período de setembro de 1999 a novembro de 2000, foram operados 22 pacientes, com idade média de 32 anos, sendo $90 \%$ do sexo feminino. As cirurgias foram assim caracterizadas: dez lipodorsoabdominoplastias com enxertia de gordura nos glúteos; nove lipodorsoabdominoplastias, três delas combinadas com mamoplastia. O retalho de deslizamento vascularizado idealizado por Avelar ${ }^{10}$, associado ao túnel cirúrgico xifoumbilical, permite a descida total do retalho abdominal e a plicatura, concretizando a lipodorsoabdominoplastia.

\section{MÉTODO}

No período de setembro de 1999 a junho de 2010, 606 pacientes, com idade média de 32 anos, foram submetidas à lipoabdominoplastia associada à lipoaspiração de flancos e dorso mais enxertia de gordura nos glúteos, caracterizando a lipodorsoabdominoplastia (Tabela 1).

Quando indicado, várias pacientes se submeteram também à mamoplastia redutora ou de aumento.

Todas as pacientes realizaram os exames de rotina, como risco cirúrgico e de prevenção de trombose venosa profunda.

As cirurgias foram realizadas por equipe treinada para que a cirurgia da mama terminasse antes ou ao mesmo tempo da lipodorsoabdominoplastia, com duração média de quatro horas.

Tabela 1 - Pacientes operadas no período de setembro de 1999 a junho de 2010 e caracterização das cirurgias

\begin{tabular}{|c|c|c|}
\hline Pacientes operados & & 606 \\
\hline \multirow{2}{*}{ Sexo } & Feminino & $100 \%$ \\
\hline & Masculino & - \\
\hline Idade & 50 anos & $\begin{array}{l}\text { Média de } \\
32 \text { anos }\end{array}$ \\
\hline \multirow{2}{*}{ Anestesia } & Peridural & $90,09 \%$ \\
\hline & Geral & $90,09 \%$ \\
\hline $\begin{array}{l}\text { Volume de } \\
\text { Gorduras aspiradas } \\
\text { em ML }\end{array}$ & $\begin{array}{c}800 \mathrm{ml} \mathrm{a} 6.910 \mathrm{ml} \text { com média } \\
\text { de } 2.524 \mathrm{ml}\end{array}$ & \\
\hline Tempo de Cirurgia & 3h30' a $4 \mathrm{~h} 30$ ' média de $4 \mathrm{~h}$ & \\
\hline \multirow{4}{*}{$\begin{array}{l}\text { Cirurgias } \\
\text { Realizadas }\end{array}$} & Lipodorsoabdominoplastia & 378 \\
\hline & $\begin{array}{l}\text { Lipodorsoabdominoplastia } \\
\text { com prótese mamária }\end{array}$ & 166 \\
\hline & $\begin{array}{l}\text { Lipodorsoabdominoplastia } \\
\text { com mamoplastia }\end{array}$ & 62 \\
\hline & Total & 606 \\
\hline
\end{tabular}


O procedimento foi indicado a pacientes dos tipos III e $\mathrm{V}$ segundo a classificação de Bozola e Psillakis ${ }^{12}$. Pacientes com excesso de peso e pós-cirurgia bariátrica não foram incluídas.

\section{Lipoaspiração do dorso}

Com a redução do tempo cirúrgico e da morbidade, foi possível acrescentar à cirurgia de lipoabdominoplastia a lipoaspiração do dorso e flancos. Desse modo, são alcançados melhores resultados, uma vez que a lipoaspiração da região abdominal se completa com a da região dorsal por retirar todo o excesso de gordura indesejável e modelar o contorno corporal, possibilitando atingir-se um nível de excelência na cirurgia e assegurar satisfação à paciente.

\section{Técnica cirúrgica}

A marcação é realizada com a paciente em pé, dandose especial atenção à posição do umbigo que pode estar em posição normal, acima ou abaixo em relação à parede abdominal, dificultando ou não a descida do retalho à região hipogástrica.

Pode-se, pela dedução de uma fórmula matemática, calcular os parâmetros de proporção de beleza, considerando a proporção ideal para o umbigo em relação ao conjunto abdominal. Para isso, são adotados como pontos de referência:

a) distância da parte mais alta do monte púbis à cicatriz umbilical (epicentro);

b) epicentro da cicatriz umbilical ao apêndice xifoide;

c) proporção de $1 / 1,5$ entre púbis e epicentro e epicentro e apêndice xifoide.

Com base nesta proporção, são nomeadas variáveis para estes pontos de referência no intuito de encontrar a posição do umbigo em lipodorsoabdominoplastias a serem realizadas.

Demonstração da fórmula:

- $\mathrm{X}=$ Distância correspondente da sínfise do púbis ao epicentro da cicatriz umbilical.

- $3 X \backslash 2=$ Epicentro da cicatriz umbilical ao apêndice xifóide.

- D = Distância total do eixo abdominal, correspondendo à distância da sínfise púbica ao apêndice xifoide.

Tomando-se por base estas variáveis, medidas individualmente em cada paciente, consegue-se chegar a uma constante, independentemente do biótipo, que é a posição ideal do umbigo em relação à sínfise púbica, ou seja, a posição X.

Demonstração da fórmula:

- $\mathrm{d}=\mathrm{x}+3 \mathrm{x} / 2$

- $\mathrm{d}=2 \mathrm{x}+3 \mathrm{x} / 2$

- $\mathrm{d}=5 \mathrm{x} / 2$

- onde $\mathrm{x}$ agora se denomina $\mathrm{U}$ - umbigo ideal

Então:

- $\mathrm{d}=5 \mathrm{U} / 2$

- $2 \mathrm{~d}=5 \mathrm{U}$

- $\mathrm{U}=2 \mathrm{~d} / 5$

Com essa fórmula, é possível chegar à posição ideal do umbigo, ou seja, 1/1,5 ou 1/1,618 de Leonardo Da Vinci ou da Divina Proporção (Figura 1).

Inicia-se a cirurgia com a paciente em posição de decúbito ventral, para a lipoaspiração da região dorsal, lombar e flancos e da região posterior; nesta posição, também se realiza a enxertia glútea, com hipercorreção de, em média, $400 \mathrm{ml}$ a $600 \mathrm{ml}$ de gordura, a qual não recebe nenhum tratamento e é injetada imediatamente após a sua retirada.

A paciente é então colocada em decúbito dorsal, quando se faz a lipoaspiração de toda a região abdominal e flancos anteriores; neste momento, inicia-se a cirurgia da mama para inclusão de próteses, quando indicada, de tal modo que o tempo cirúrgico coincida com o da fase abdominal.

Após a lipoaspiração do abdome, a incisão é feita na região hipogástrica, segundo a marcação da técnica de Callia ${ }^{5}$, descolando-se o retalho no sentido cranial até a região do umbigo. Este é isolado e decide-se sobre a sua desinserção ou não, dependendo se será excisado parcial ou totalmente ${ }^{13}$.

Procede-se, então, à confecção do túnel cirúrgico xifopubiano e plicatura dos músculos retoabdominais ${ }^{14}$.

Dependendo da posição do umbigo (normal, alto ou baixo) e da quantidade de gordura, o retalho infra-abdominal poderá descer parcial ou totalmente com a desinserção do umbigo cerca de $2 \mathrm{~cm}$ abaixo de sua posição original ou, no caso de sua não desinserção, este permanecerá no local de origem quando da retirada de todo o retalho inferior abdominal, sendo reposicionado na parede abdominal. Antes do reposicionamento do retalho abdominal remanescente na região hipogástrica, realiza-se a lipectomia da gordura excedente após a lipoaspiração. Esta lipectomia é feita abaixo da fáscia de Scarpa para manter a viabilidade vascular.

Os seguintes cuidados são tomados: uso de meias elásticas e aparelho de compressão intermitente por 24 horas e infiltração da área a ser tratada por lipoaspiração com solução de adrenalina em soro fisiológico a $0,9 \%$ na proporção de 1:500.00ui (Figuras 2 a 5).

\section{RESULTADOS}

A lipodorsoabdominoplastia tem se mostrado um procedimento seguro e de pequena morbidade quando se observam os devidos cuidados como:

- rigoroso pré-operatório, indicação cirúrgica seletiva e observação do tempo de duração da cirurgia em torno de quatro horas;

- equipe treinada, deambulação precoce, aplicação de massagem linfática, uso de macaquinhos e tala cirúrgica, uso de clexane $40 \mathrm{mg}$ para prevenir a trombose ${ }^{15}$.

No pós-operatório, verificou-se um contorno corporal de silhueta natural, com evidência para cinturas e nádegas, resultados que geraram satisfação para equipe cirúrgica e pacientes.

No período estudado, a complicação mais comum foi o seroma, com índice de $10 \%$, que diminuiu para até $0 \%$ com a utilização dos pontos de Baroudi e Ferreira? ${ }^{9}$. 


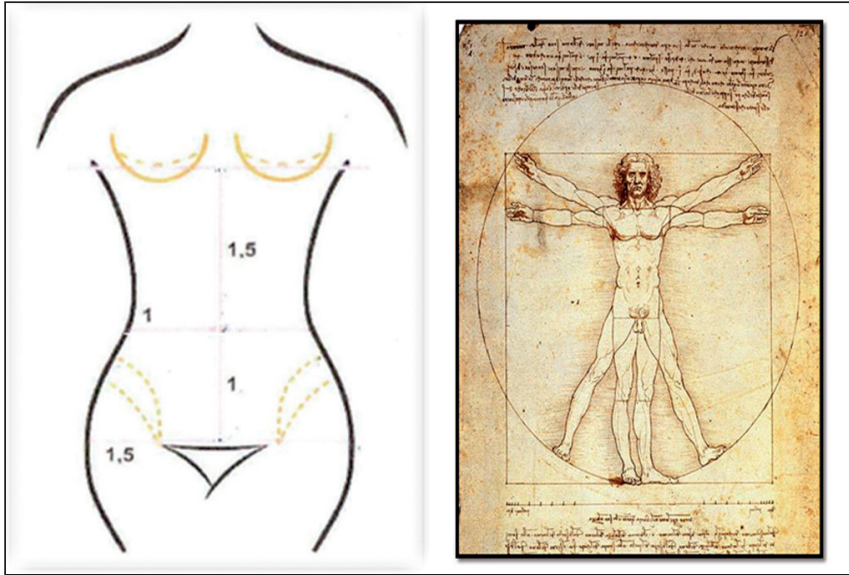

Figura 1 - Homem Vitruviano: Vitrúvio tentou encaixar as proporções do corpo humano dentro da figura de um quadrado e de um círculo, mas foi com Leonardo da Vinci que o encaixe

saiu corretamente. Proporção ideal do umbigo na parede abdominal - $1 / 1$.
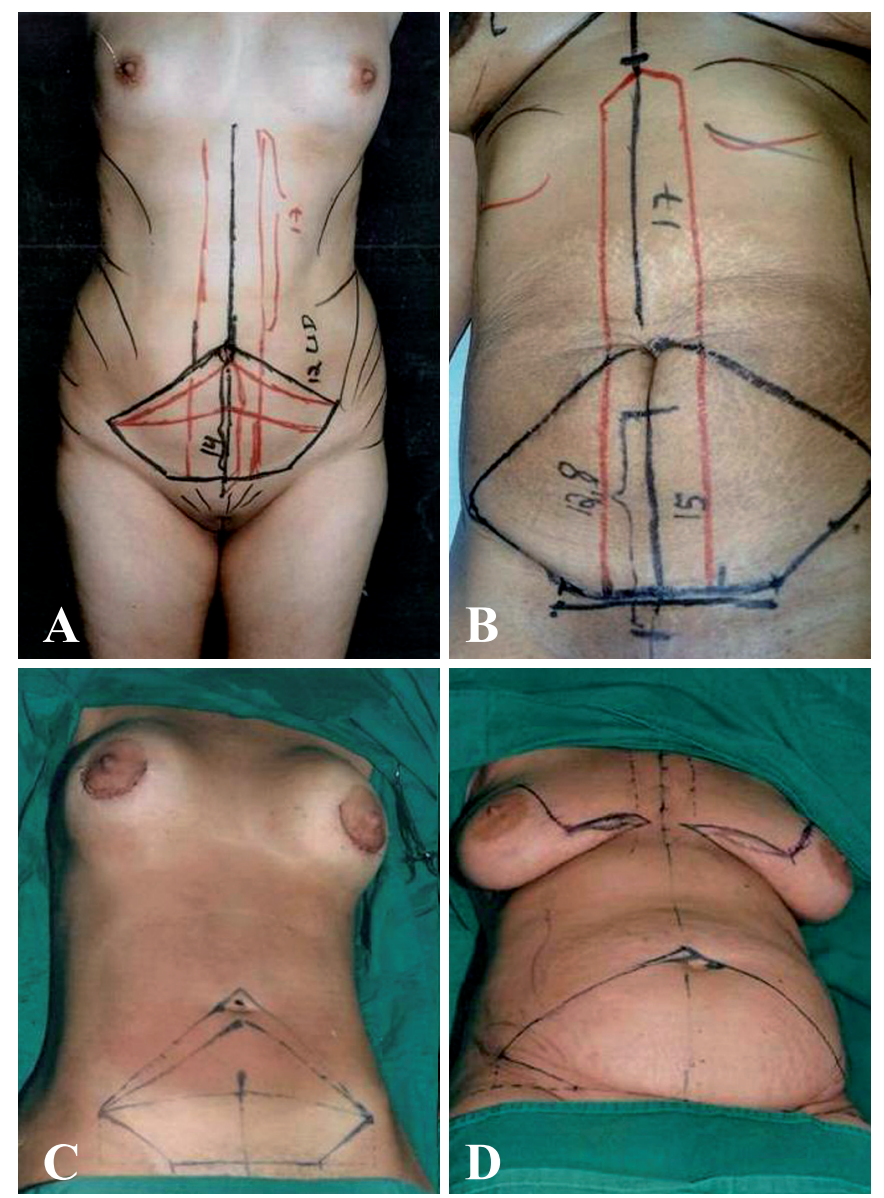

Figura 2 - Marcação: especial atenção à posição do umbigo e qualidade da pele que facilita ou não a descida do retalho abdominal à região hipogástrica, resultando em cicatrizes menores, Tinvertido ou cicatriz ampla.
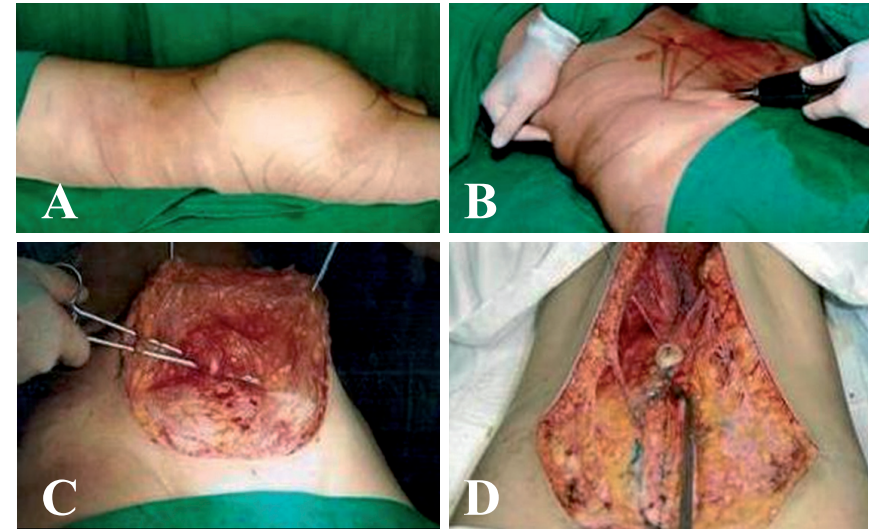

Figura 3 - Inicia-se o procedimento com a lipoaspiração das costas e flancos e, posteriormente, da região ventral, que se unem e fornecem gordura para a enxertia glútea. A incisão do abdome é realizada no sentido cranial, com desinserção ou não do umbigo. $A$ plicatura dos músculos reto abdominais é obrigatória.
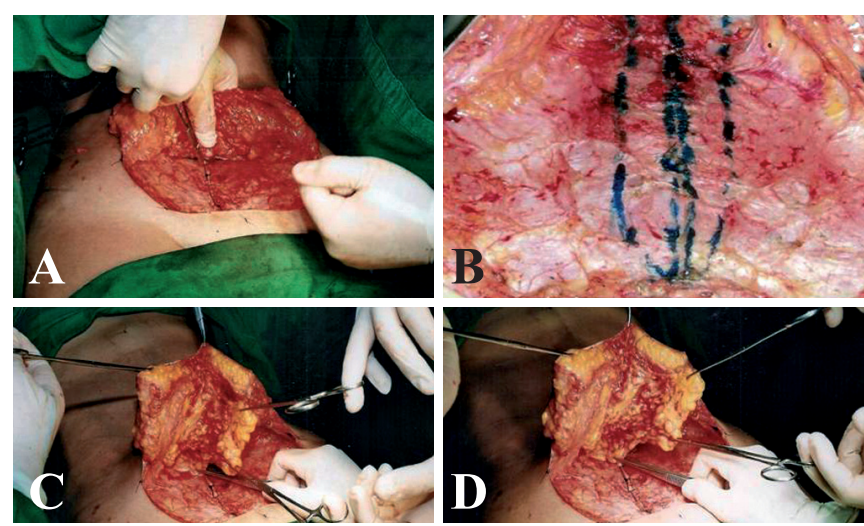

Figura 4 - Reposicionamento do umbigo em média 2,0 cm abaixo de sua posição de origem. Marcação da plicatura dos músculos reto abdominais e lipectomia da gordura excedente abaixo da fáscia de Scarpa, após a lipoaspiração da camada lamelar.
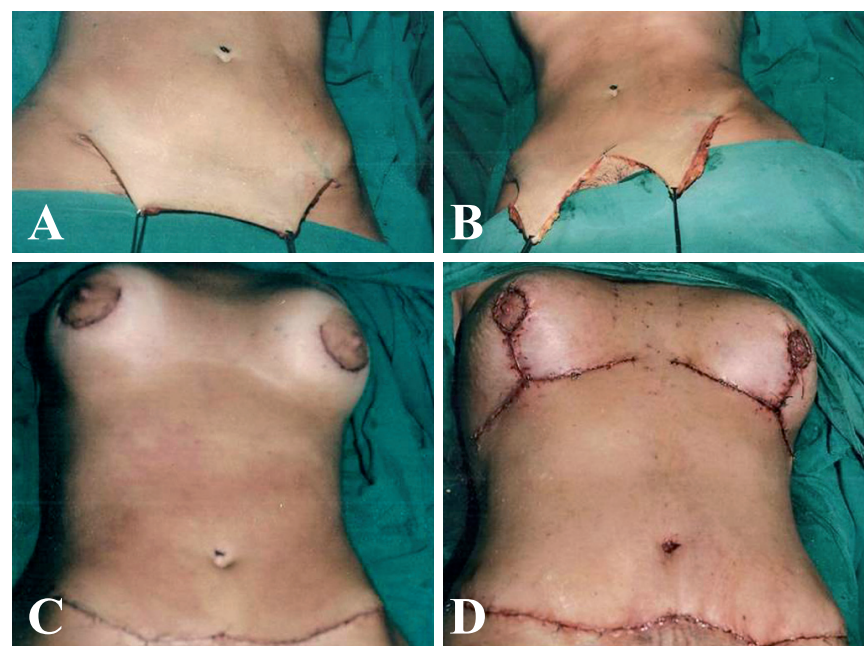

Figura 5 - Retalhos abdominais a serem retirados com e sem desinserção do umbigo e pós-operatório imediato. 
Entre as complicações citadas na literatura, ocorreram: uma infecção, uma deiscência por infecção localizada no abdome e duas necroses de pele, uma em paciente fumante e outra em abdome com cicatriz pararretal direita, externa e extensa (Figuras 6 a 9).
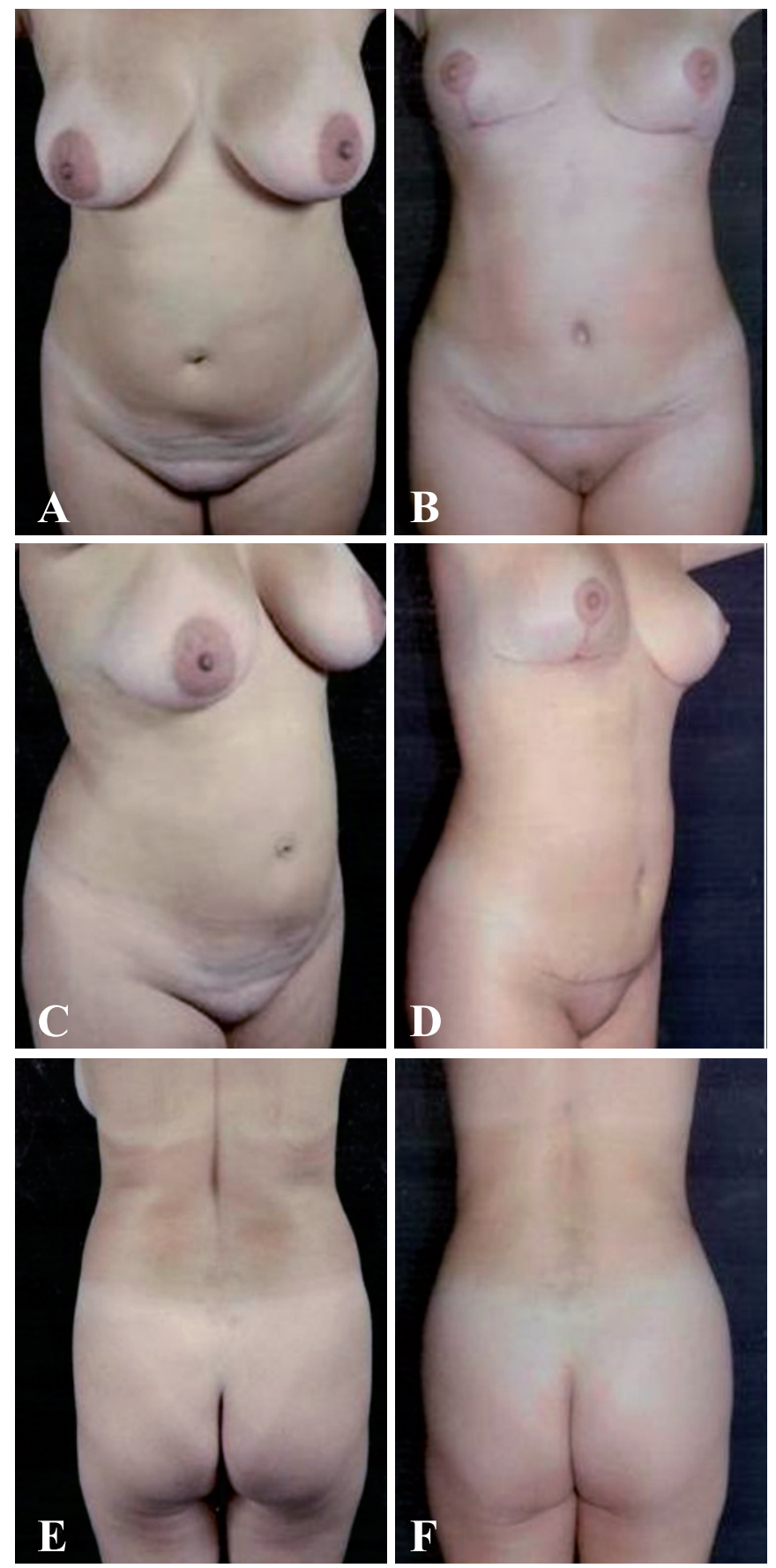

Figura 6-Fotografias pré (A, C e E) e pós-operatórias (B, D e F) de paciente submetida a lipodorsoabdominoplastia com enxertia glútea e mamoplastia redutora.
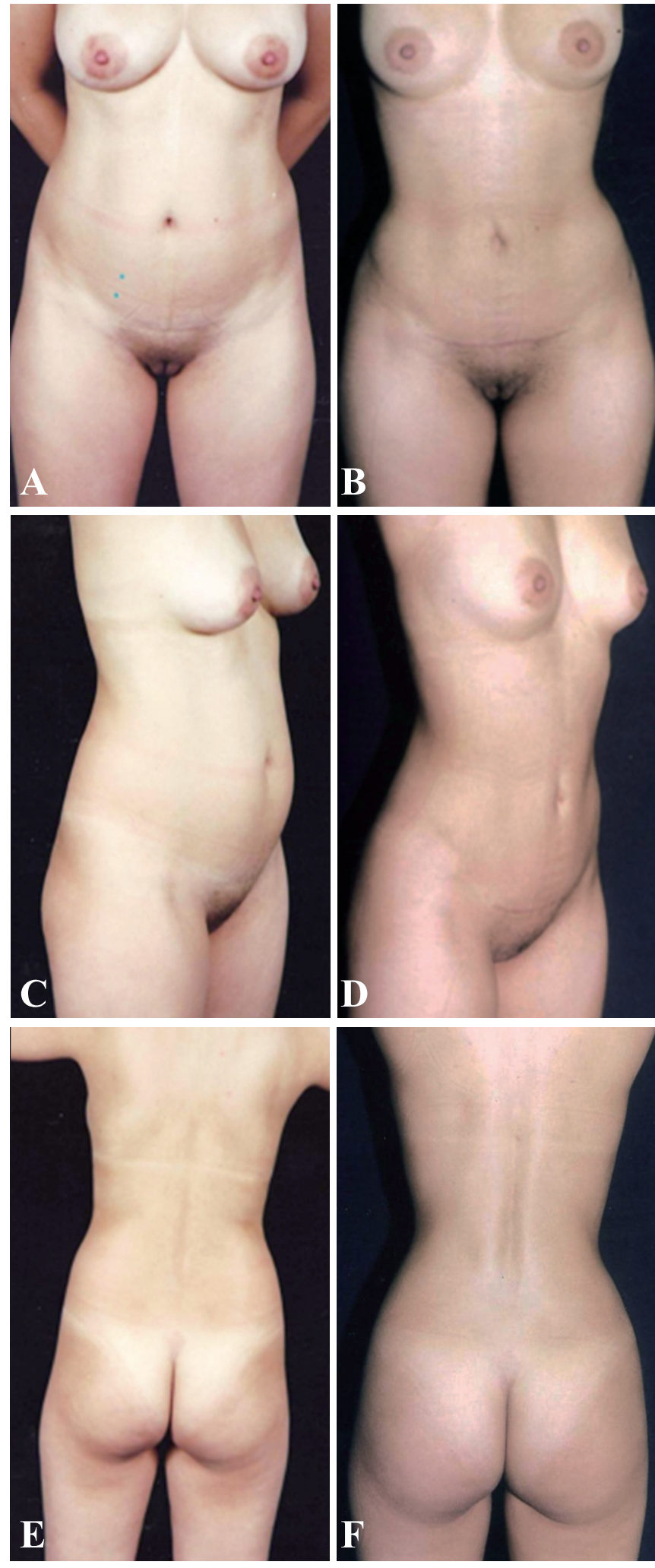

Figura 7-Fotografias pré (A, C e E) e pós-operatórias (B, De $F)$ de paciente submetida somente a lipodorsoabdominoplastia e enxertia glútea. 

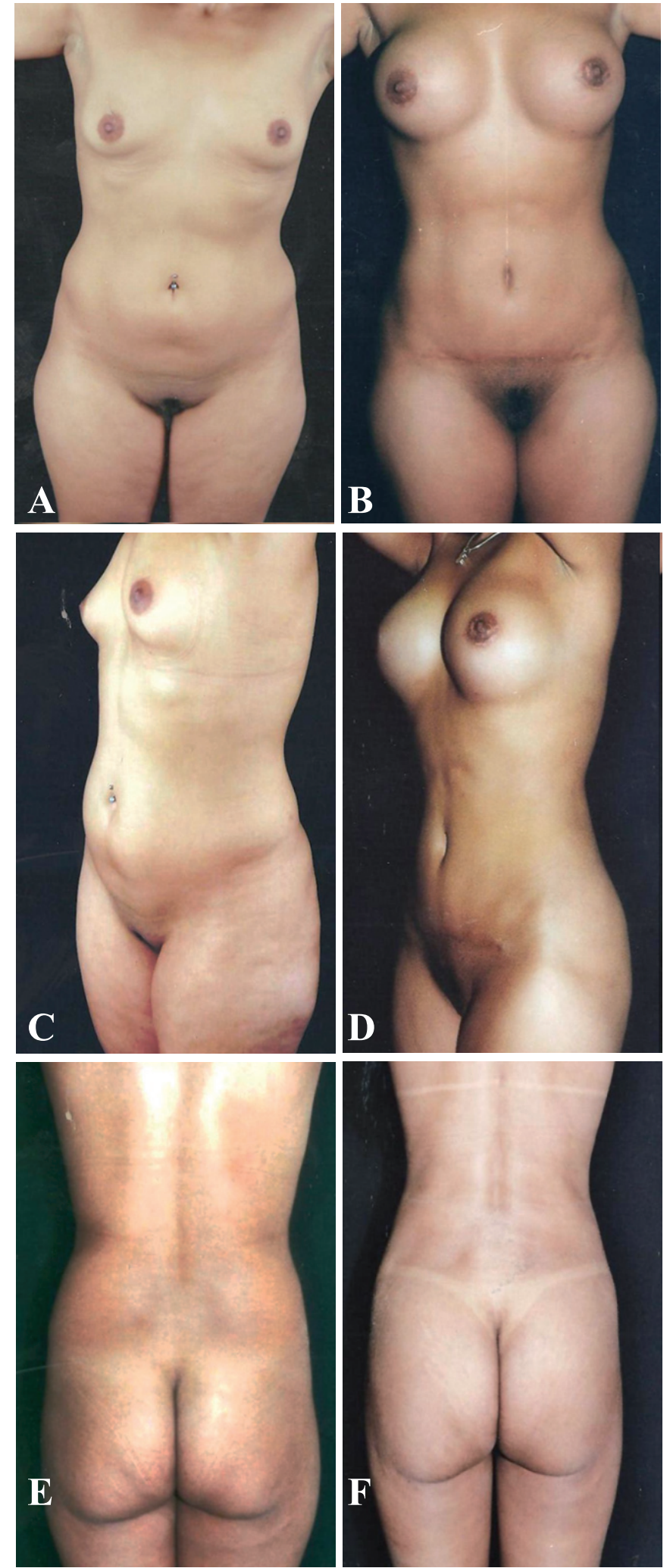

Figura 8-Fotografias pré (A, C e E) e pós-operatórias ( $B, D$ e F) de paciente submetida à lipodorsoabdominoplastia, enxertia glútea e implante de prótese de silicone.
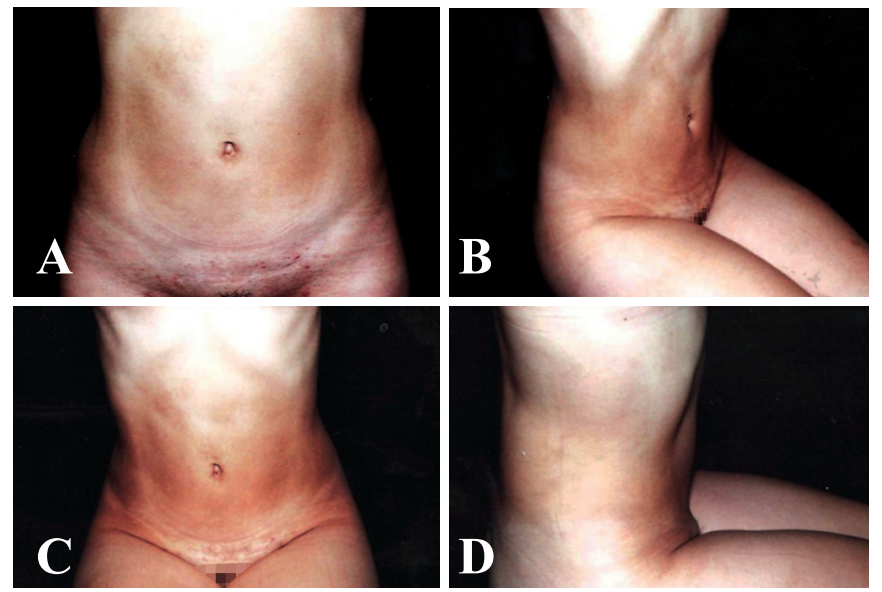

Figura 9 - Pós-operatório de paciente sentada após um ano.

\section{DISCUSSÃO}

Denomina-se lipodorsoabdominoplastia a cirurgia em que a lipoaspiração do dorso e flancos complementa a lipoaspiração da parede abdominal, fornecendo gordura para a enxertia glútea. A boa vascularização viabiliza os retalhos cutâneos com menor risco de epiteliose, necrose ou deiscência. $\mathrm{O}$ procedimento exige equipe treinada, com maior curva de aprendizado e tempo cirúrgico em torno de quatro horas, uma vez que a cirurgia das mamas termina ao mesmo tempo que a do abdome. A seleção criteriosa de pacientes é preponderante.

\section{CONCLUSÃO}

A lipodorsoabdominoplastia é a evolução cirúrgica natural da abdominoplastia clássica acrescida da lipoaspiração em toda a parede abdominal e do túnel cirúrgico xifoumbilical, o que possibilita a lipoaspiração dos flancos e dorso e a plicatura dos músculos reto abdominais, proporcionando resultados com nível de excelência e satisfação para cirurgiões e pacientes.

\section{REFERÊNCIAS}

1. Kelly HA. Excision of the fat abdominal wal lipectomy. Surg Gynecol Obstetr. 1910; 10: 229.

2. Babcock W. The correction of the obese and relaxed abdominal wall with special reference to the use buried silver chain. Am J Obstr. 1916;74:596-611.

3. Thorek M. Plastic surgery of the breast and abdominal wall. Springfield: Charles Thomas; 1942.

4. Pontes R. Plástica abdominal: importância de sua associação à correção das hérnias incisionais. Rev Bras Cir. 1965;52(2):85-92.

5. Callia W. Contribuição para o estudo da correção do abdome pêndulo e globono - técnica original [Tese de Doutorado]. São Paulo: Faculdade de Medicina da USP;1965. 
6. Pitanguy I. Surgical reduction of the abdomen, thighs and buttocks. Surg Clin North Am. 1971;51(2):479-89.

7. Sinder R. Plastic surgery of the abdome: técnica pessoal de abdominoplastia, com prévio descolamento de retalho supra-umbilical e uso de retalho dermoadiposo. In: VI International Congress of Plastic and Reconstrutive Surgery; 1975 Ago 25; Paris, França.

8. Illouz YG. Une nourvelle technique pour leslipodystrophies localesées. Rev Chir Esther. 1980;4:19.

9. Baroudi R, Ferreira CA. Seroma: how to avoid it and how to treat it. Aesthet Surg J. 1998;18(6):439-41.

10. Avelar JM. Abdominoplasty: a new technique without undermining and fat layer removal. Arq Catarin Med. 2000; 29:147-9.

11. Leão C. Abdominoplastia: uma nova abordagem. In: Anais do XXXVII
Congresso Brasileiro de Cirurgia Plástica; 2000 nov 11-15; Porto Alegre-RS, Brasil.

12. Bozola AR, Psillakis JM. Abdominoplasty: a new concept and classification for treatment. Plast Reconstr Surg. 1988;82(6):983-93.

13. Leão C. Curso de Abdominoplastia. Chair man: AR Bozola: In: Anais do XXXVII Congresso Brasileiro de Cirurgia Plástica; 2000 nov 11-15; Porto Alegre-RS, Brasil.

14. Jaimovich CA, Mazzarone F, Parra JFN, Pitanguy I. Semiologia da parede abdominal: seu valor no planejamento da abdominoplastia. Rev Soc Bras Cir Plast. 1999;14(3):21-50.

15. Anger J, Baruzzi ACA, Knobel E. Um protocolo de prevenção de trombose venosa profunda em cirurgia plástica. Rev Soc Bras Cir Plast. 2003;18(1):51-4.

Correspondência para:

Célio Leão

Rua T-37, 2726 - Setor Bueno - Goiânia, GO, Brasil - CEP 74230-020

E-mail: celioleao1@hotmail.com 